\title{
Comparison of Magnetic Resonance Enterography Findings and Clinical Index of the Disease Activity Score in Crohn's Disease
}

Authors: Karolina Siejka ${ }^{1}$, Maryla Kuczyńska ${ }^{1}$, Małgorzata Nowakowska ${ }^{1}$, Monika Piekarska ${ }^{1}$, Monika Zbroja $^{1}$, Weronika Cyranka ${ }^{1}$, Magdalena Grzegorczyk ${ }^{1}$, Anna Drelich-Zbroja ${ }^{1}$ (mentor), Paulina Furtak ${ }^{2}$ (mentor)

${ }^{1}$ Medical Univesity of Lublin, Department of Interventional Radiology and Neuroradiology, Medical University of Lublin, Poland, Lublin, Poland,

${ }^{2}$ Medical Univesity of Lublin, Department of Gastroenterology with the Endoscopic Laboratory, Independent Public Teaching Hospital No. 4 in Lublin, Poland, Lublin, Poland

DOI: https://doi.org/10.26800/LV-142-supp5-54

\section{Introduction}

Crohn's disease $(C D)$ is a chronic inflammatory disease of the gastrointestinal tract. It usually affects younger people, with the peak of illness between the age of 15 and 25. MR enterography (MRE) is a proven and reliable modality in the evaluation of the lesion extent within this group of patients.

\section{Aim}

The purpose of the study was to compare MRE findings with clinical index of the disease activity CDAl score, in order to verify the utility of MRE in the precise assessment of disease severity.

\section{Materials/Methods}

46 consecutive patients underwent MRE on a $1.5 \mathrm{~T}$ scanner according to a standard protocol. All studies were performed in a prone position, using mannitol as a positive oral contrast agent. CDAI score was assessed prior to examination by a specialist in the field of gastroenterology.

\section{Results}

32 patients had multifocal lesions, with the majority $(\mathrm{N}=23)$ localized within both small and large intestine. 14 patients presented with unilocular lesions, 14 patients had a history of previous bowel resection due to disease complications. Penetrating disease was diagnosed in 15 subjects. The results indicate a good correlation between MRE and CDAl assessment in groups of patients with moderate and severe clinical symptoms. However, there is a significant discrepancy in the group of patients with mild disease activity according to CDAI. Out of 23 patients with clinically mild symptoms, 1 exhibited MRE characteristics of severe, 9 of moderate, 7 of mild inflammatory intestinal lesions and 6 of no active lesions.

\section{Conclusion}

There is good accordance between MRE and clinical findings in patients with prominent disease symptoms, indicating that enterography might be a promising modality in terms of lesion activity grading. Nonetheless, there exist a significant group of patients with mild disease symptoms, in whom MRE tends to show full spectrum of disease severity.

Keywords: Crohn's disease, inflammatory bowel disease, MRE 